Supplement of Atmos. Chem. Phys., 18, 7489-7507, 2018

https://doi.org/10.5194/acp-18-7489-2018-supplement

(C) Author(s) 2018. This work is distributed under

the Creative Commons Attribution 4.0 License.

(c) (1)

Supplement of

\title{
Impacts of biogenic and anthropogenic emissions on summertime ozone formation in the Guanzhong Basin, China
}

Nan Li et al.

Correspondence to: Nan Li (linan@ nuist.edu.cn)

The copyright of individual parts of the supplement might differ from the CC BY 4.0 License. 


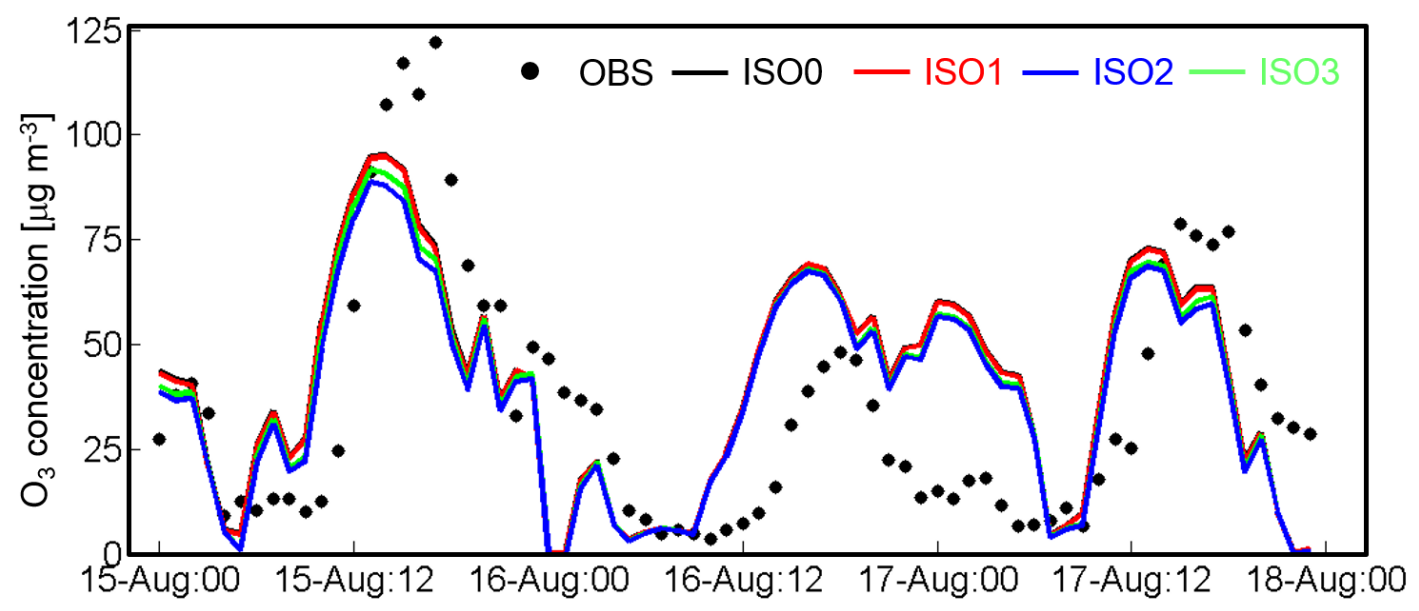

Figure S1. $\mathrm{O}_{3}$ concentrations changes due to updated isoprene chemistry at $\mathrm{Xi}$ 'an Jiaotong University for the period of $15^{\text {th }}$ to $17^{\text {th }}$ August 2011 . 


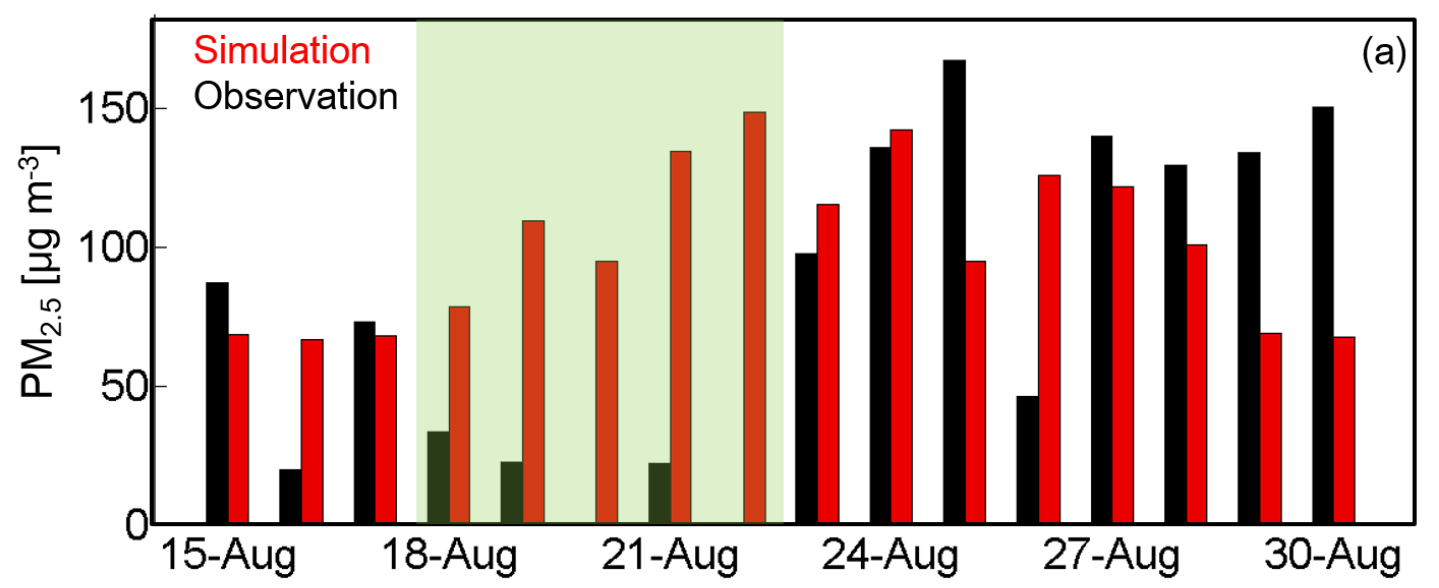

(b) Simulated $\mathrm{PM}_{2.5}$

(c) Observed $\mathrm{PM}_{2.5}$

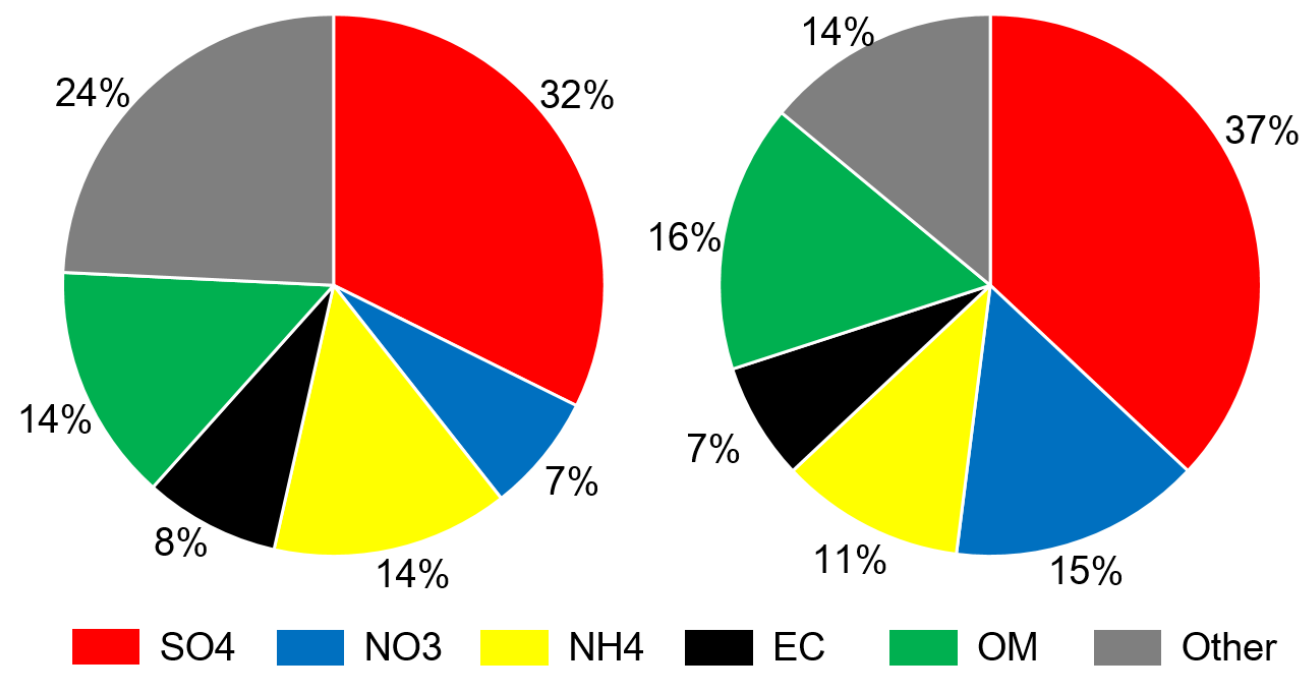

Figure S2. Observed and simulated $\mathrm{PM}_{2.5}$ concentrations (a) and compositions (b and c) at Xi'an Jiaotong University in August 2011. The components are calculated during the periods excluding the rainy days (the green shadow). 


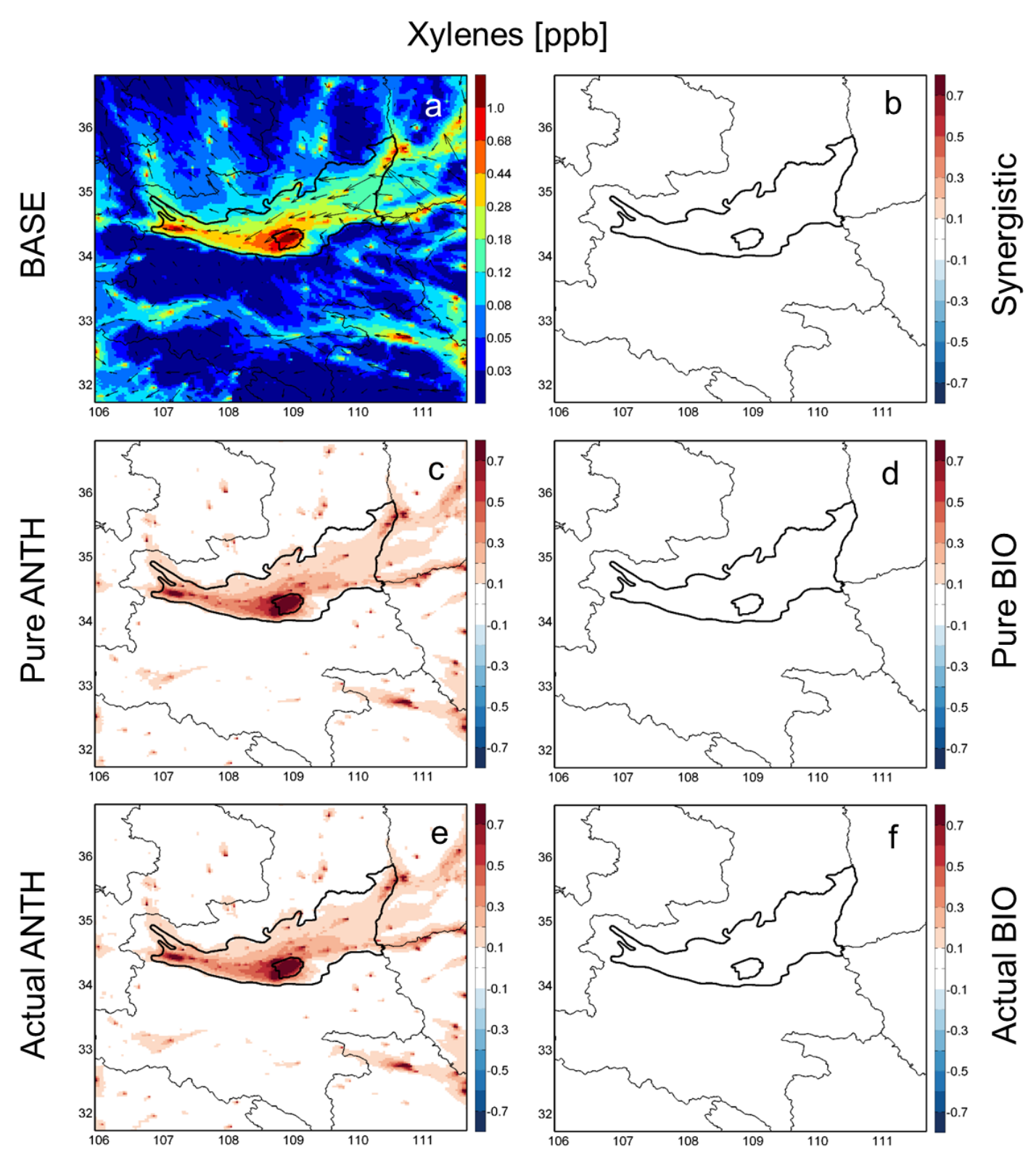

Figure S3. Spatial distributions of monthly mean concentrations of xylenes in August 2011. (a) is the result from the BASE simulation, overlaid with simulated wind vectors. (b)-(f) are simulated xylenes concentrations contributed from synergistic anthropogenic and biogenic, pure anthropogenic, pure biogenic, actual anthropogenic and actual biogenic sources, respectively. 


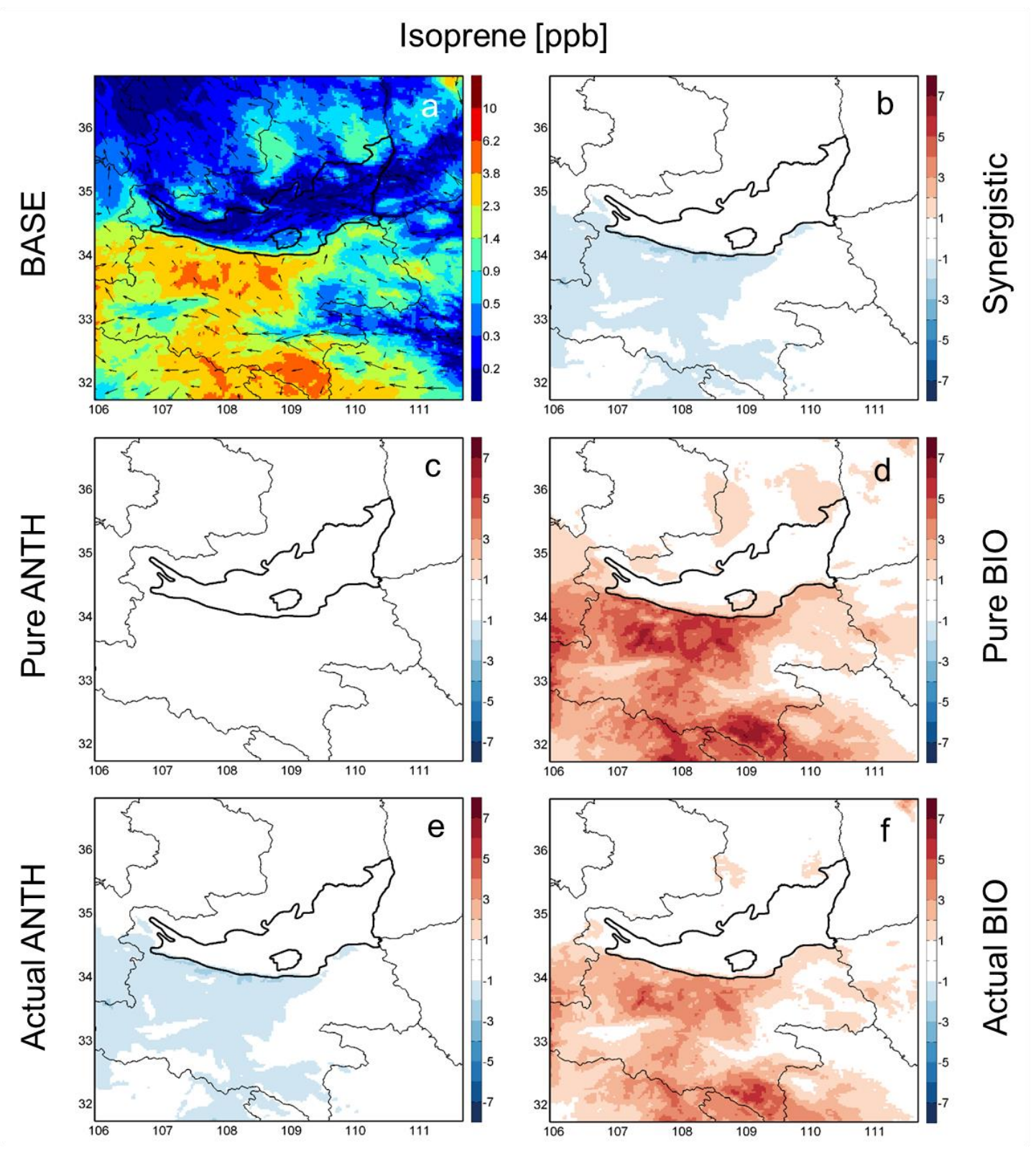

Figure S4. Spatial distributions of monthly mean concentrations of isoprene in August 2011. (a) is the result from the BASE simulation, overlaid with simulated wind vectors. (b)-(f) are simulated isoprene concentrations contributed from synergistic anthropogenic and biogenic, pure anthropogenic, pure biogenic, actual anthropogenic and actual biogenic sources, respectively. 

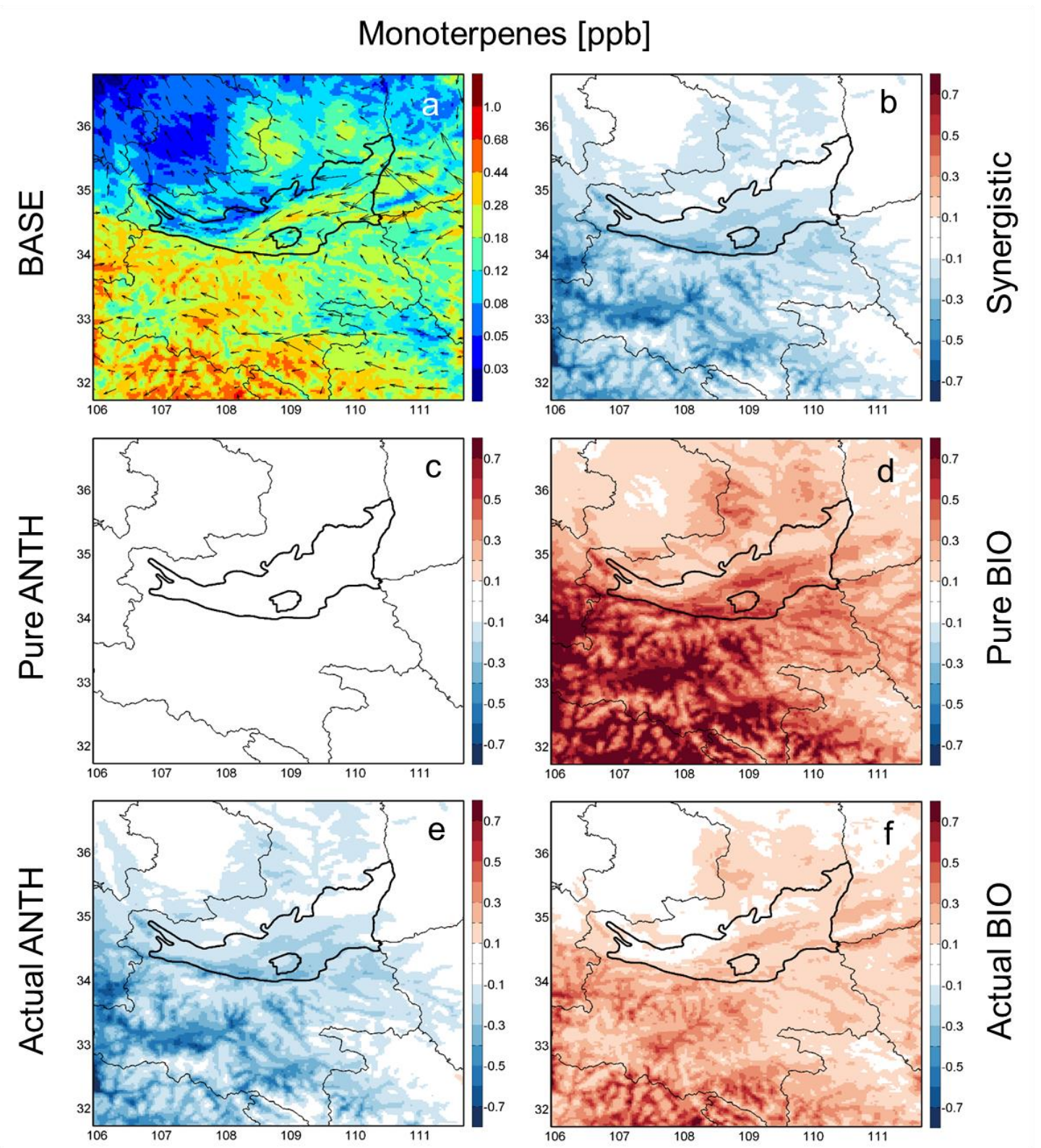

Figure S5. Spatial distributions of monthly mean concentrations of monoterpenes in August 2011. (a) is the result from the BASE simulation, overlaid with simulated wind vectors. (b)-(f) are simulated monoterpenes concentrations contributed from synergistic anthropogenic and biogenic, pure anthropogenic, pure biogenic, actual anthropogenic and actual biogenic sources, respectively. 


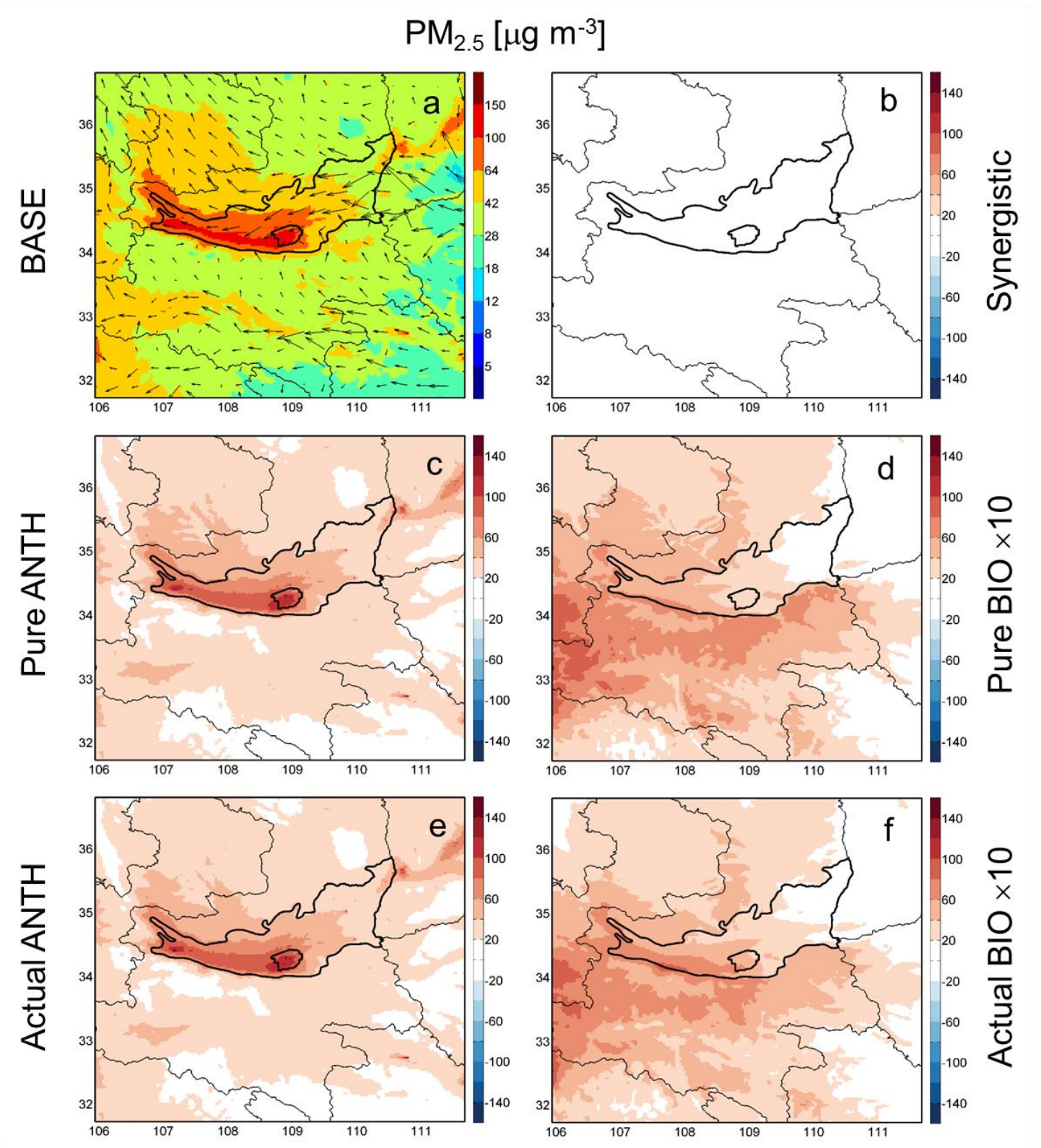

Figure S6. Spatial distributions of monthly mean concentrations of $\mathrm{PM}_{2.5}$ in August 2011. (a) is the result from the BASE simulation, overlaid with simulated wind vectors. (b)-(f) are simulated $\mathrm{PM}_{2.5}$ concentrations contributed from synergistic anthropogenic and biogenic, pure anthropogenic, pure biogenic, actual anthropogenic and actual biogenic sources, respectively. 


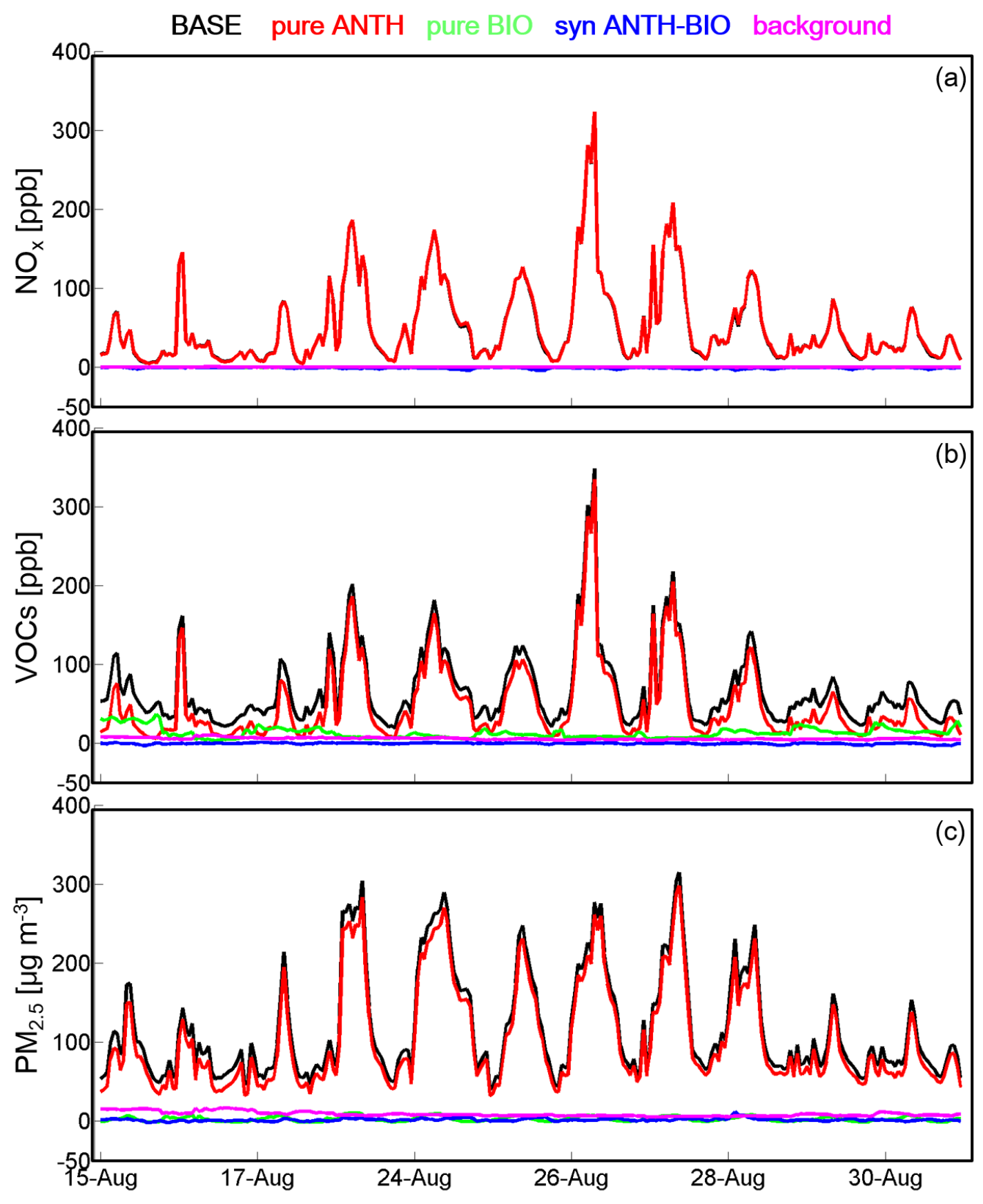

Figure S7. Temporal patterns of the simulated concentrations of $\mathrm{NO}_{\mathrm{x}}$, VOCs and $\mathrm{PM}_{2.5}$ and the various contribution components during the period from $15^{\text {th }}$ to $30^{\text {th }}$ August 2011, excluding the rainy days $\left(18^{\text {th }}-22^{\text {nd }}\right.$ August $)$. 
Table S1. Updated isoprene oxidation chemistry (unit for reaction rate is molecule $\mathrm{cm}^{-1} \mathrm{~s}^{-1}$ )

\begin{tabular}{|c|c|c|}
\hline \multicolumn{2}{|l|}{ Reactions } & Reaction rates \\
\hline \multicolumn{2}{|l|}{ ISOPO2+NO > } & $2.7 \times 10^{-12}$ \\
\hline \multicolumn{2}{|c|}{$\mathrm{ALD}+\mathrm{HCHO}+\mathrm{HO} 2+0.96 * \mathrm{NO} 2+0.04 * \mathrm{ISOPN}$} & $\times \exp (350 / \mathrm{T})$ \\
\hline \multirow{2}{*}{\multicolumn{2}{|c|}{$\begin{array}{l}\text { ISOPO } 2> \\
2 * \mathrm{HO} 2+\mathrm{HCHO}+0.33 * \mathrm{MGLY}+0.5 * \mathrm{GLYALD}+0.25 * \mathrm{GLYX}+1.5 * \mathrm{HACET}\end{array}$}} & $4.07 \times 10^{8}$ \\
\hline & & $\times \exp (-7694 / \mathrm{T})$ \\
\hline Species name & \multicolumn{2}{|l|}{ Description } \\
\hline $\mathrm{T}$ & \multicolumn{2}{|l|}{ Temperature (K) } \\
\hline $\mathrm{NO}$ & \multicolumn{2}{|l|}{ Nitric oxide } \\
\hline NO2 & \multicolumn{2}{|l|}{ Nitrogen dioxide } \\
\hline $\mathrm{HO} 2$ & \multicolumn{2}{|l|}{ Hydroperoxy radical } \\
\hline ISOPO2 & \multicolumn{2}{|c|}{ Hydroperoxy radicals from isoprene oxidation by $\mathrm{OH}$} \\
\hline ISOPN & \multicolumn{2}{|l|}{ Organic nitrate } \\
\hline $\mathrm{HCHO}$ & \multicolumn{2}{|l|}{ Formaldehyde } \\
\hline ALD & \multicolumn{2}{|l|}{ Acetaldehyde and higher aldehydes } \\
\hline HACET & \multicolumn{2}{|l|}{ Hydroxyacetone } \\
\hline MGLY & \multicolumn{2}{|l|}{ Methylglyoxal } \\
\hline GLYALD & \multicolumn{2}{|l|}{ Glycolaldehyde } \\
\hline GLYX & \multicolumn{2}{|l|}{ Glyoxal } \\
\hline
\end{tabular}


Table S2. $\mathrm{O}_{3}$ concentration changes due to updated isoprene chemistry averaged for urban Xi'an

\begin{tabular}{lccc}
\hline $\mathrm{O}_{3}$ concentration $(\mathrm{ppb})$ & $\mathrm{ISO}^{\mathrm{b}}{ }^{\mathrm{b}}-\mathrm{ISO}{ }^{\mathrm{a}}$ & $\mathrm{ISO}^{\mathrm{c}}{ }^{\mathrm{c}}$-ISO0 & $\mathrm{ISO}^{{ }^{\mathrm{d}}-\mathrm{ISO} 0}$ \\
\hline Daily peak & -0.47 & -4.6 & -2.9 \\
$24 \mathrm{~h}$ average & -0.27 & -2.9 & -2.0 \\
\hline
\end{tabular}

${ }^{\text {a }}$ ISO0: simulation using standard RADM2 mechanism

${ }^{\mathrm{b}}$ ISO1: same as ISO0, but add isomerization of radicals from isoprene oxidation by $\mathrm{OH}$

${ }^{\mathrm{c}}$ ISO2: same as ISO0, but add formation of hydroxynitrates

${ }^{\mathrm{d}}$ ISO3: same as ISO0, but with revisions in both ISO1 and ISO2. 
Table S3. Domain-wide amount of emissions in August 2011

\begin{tabular}{lccc}
\hline & Anthropogenic $\left(\mathrm{Gg} \mathrm{mon}^{-1}\right)$ & Biogenic $\left(\mathrm{Gg} \mathrm{mon}^{-1}\right)$ & Total $\left(\mathrm{Gg} \mathrm{mon}^{-1}\right)$ \\
\hline $\mathrm{SO}_{2}$ & $358( \pm 31 \%)^{\mathrm{a}}$ & - & 358 \\
$\mathrm{NO}_{\mathrm{x}}$ & $110( \pm 37 \%)^{\mathrm{a}}$ & 2.1 & 112 \\
$\mathrm{NH}_{3}$ & $69.0( \pm 153 \%)^{\mathrm{a}}$ & - & 69.0 \\
$\mathrm{PM}_{2.5}$ & $163( \pm 133 \%)^{\mathrm{a}}$ & - & 163 \\
$\mathrm{VOCs}$ & $72.2( \pm 78 \%)^{\mathrm{a}}$ & 204 & 276 \\
\multicolumn{1}{c}{ Isoprene } & $<0.1$ & 157 & 157 \\
Monoterpenes & - & 22.8 & 22.8 \\
Alkanes & 34.4 & 5.4 & 39.8 \\
Alkenes & 21.3 & 4.9 & 26.2 \\
Aromatic & 11.5 & - & 11.5 \\
Carbonyls & 4.5 & 12.1 & 16.6 \\
Organic acids & 0.5 & 1.5 & 2.0 \\
\hline
\end{tabular}

${ }^{\mathrm{a}}$ Uncertainty in emission estimates (95\% confidence intervals). 
Table S4. Simulated $\mathrm{O}_{3}$ and VOC concentrations in urban Xi'an during $15^{\text {th }}-17^{\text {th }}$ August 2011.

\begin{tabular}{lccc}
\hline \multicolumn{1}{c}{ Species [ ppb ] } & VOC0 $^{\mathrm{a}}$ & VOC1 $^{\mathrm{b}}$ & VOC2 $^{\mathrm{c}}$ \\
\hline $\mathbf{O}_{\mathbf{3}}$ & $\mathbf{4 0 . 7}$ & $\mathbf{4 1 . 9}$ & $\mathbf{3 9 . 8}$ \\
\hline Ethane & 13.1 & 18.7 & 9.5 \\
C>2 alkanes & 11.2 & 16.1 & 8.9 \\
HCHO & 7.4 & 5.5 & 5.0 \\
Acetaldhyde & 5.4 & 4.7 & 4.2 \\
Toluene & 2.9 & 4.3 & 2.2 \\
Ethene & 2.8 & 4.2 & 2.3 \\
Organic nitrogen & 2.7 & 1.7 & 1.5 \\
Organic peroxides & 2.1 & 2.0 & 1.9 \\
C>2 alkenes & 2.0 & 3.2 & 1.7 \\
Ketones & 1.6 & 1.6 & 1.2 \\
Xylenes & 1.2 & 1.9 & 0.8 \\
Organic acids & 1.0 & 1.2 & 1.1 \\
Total VOC & $\mathbf{5 3 . 3}$ & $\mathbf{6 4 . 9}$ & $\mathbf{4 0 . 3}$ \\
\hline
\end{tabular}

${ }^{\mathrm{a}}$ VOC0: Base simulation using emission estimates from MEIC.

${ }^{\mathrm{b}}$ VOC1: Same as Base, but with an increase of anthropogenic VOC emission by $50 \%$

${ }^{\mathrm{c}}$ VOC2: Same as Base, but with an decrease of anthropogenic VOC emission by $33 \%$ 